\title{
INTEGRAL \& RXTE View of Gamma-ray Binaries
}

\author{
Jian $\mathbf{L i}^{*}$ \\ Institute of High Energy Physics, Chinese Academy of Sciences, China \\ Institut de Ciències de l'Espai (IEEC-CSIC), Spain \\ E-mail: Gianlidihep.ac.cn
}

Diego F. Torres

Institut de Ciències de l'Espai (IEEC-CSIC), Spain

Institució Catalana de Recerca i Estudis Avançats (ICREA)

E-mail: dtorresdieec.uab.es

\section{Shu Zhang}

Institute of High Energy Physics, Chinese Academy of Sciences, China E-mail: szhangdihep.ac.cn

\section{Jianmin Wang}

Institute of High Energy Physics, Chinese Academy of Sciences, China

National Astronomical Observatories of China, Chinese Academy of Sciences, China

E-mail: wangjmeihep.ac.cn

Gamma-ray binaries are X-ray binaries with gamma-ray emissions. Their multi-wavelength emissions range from radio, optical, $\mathrm{X}$-ray and to very high energy $(\mathrm{TeV}) . \mathrm{X}$-ray emissions are crucial to understand the nature of gamma-ray binaries. INTEGRAL and RXTE have covered and monitored most of the gamma-ray binaries in hard and soft X-rays. Here we report the results of several gamma-ray binaries and possible gamma-ray binaries from INTEGRAL and RXTE.

"An INTEGRAL view of the high-energy sky (the first 10 years)" 9th INTEGRAL Workshop and celebration of the 10th anniversary of the launch,

October 15-19, 2012

Bibliotheque Nationale de France, Paris, France

\footnotetext{
* Speaker.
} 


\section{Introduction}

Various classes of gamma-ray sources have been detected up to the TeV band. Among them, the class of gamma-ray emitting binaries focuses great attention. They are X-ray binaries hosting O/B companions, which have gamma-ray emission up to High-Energy (HE, E >100 MeV) and/or Very High-Energy (VHE, E $>100 \mathrm{GeV}$ ), modulated on the orbital period. Only a handful of such binaries are known (e.g., LS 5039, LSI +61 303, PSR B1259-63, HESS J0632+057, 1FGL J1018.6-5856, or Cyg X-3), although a larger population is expected. To understand their nature and particle acceleration mechanisms, X-ray emissions of gamma-ray binaries are crucial. INTEGRAL and RXTE have observed and monitored most of the gamma-ray binaries in hard and soft X-ray. Here we present the results for several confirmed (LS I +61 303, 1FGL J1018.6-5856 and HESS J0632+057) and possible gamma-ray binaries (AGL J1037-5708 and AGL J2241+4454 ) in the view of INTEGRAL and RXTE.

\section{Super-orbital modulation of LS I +61 303}

Our data set includes 473 RXTE/PCA pointed observations from 2007 August 28 to 2011 September 15 . The analysis is performed using the standard RXTE/PCA criteria. Only PCU2 has been used for the analysis. Our count rate values are given for an energy range of 3-30 keV. In order to remove the influence of several kilosecond-long flares, we cut all observations that presented a larger count rate than three times the average.

Given a six-month time bin, we take the peak X-ray flux in orbital lightcurve and compute the modulated flux fraction. The latter is defined as $\left(c_{\max }-c_{\min }\right) /\left(c_{\max }+c_{\min }\right)$, where $c_{\max }$ and $c_{\min }$ are the maximum and minimum count rates in the $3-30 \mathrm{keV}$ orbital lightcurve of that period. Results are shown in Figure 1. Table 1 presents the values of the reduced $\chi^{2}$ for fitting different models to the modulation fraction and the peak flux in X-rays. It compares the results of fitting a horizontal line, a linear fit, and two sinusoidal functions. One of the latter has the same period and phase of the radio modulation (from Gregory 2002, labeled as "Radio" in Table 1, dotted line in Figure 1). The other sine function has the same period as in radio but allowing for a phase shift from it (a solid line in Figure 1, labeled as "Shifted" in Table 1). It is clear that there is variability in the data and the sinusoidal description with a phase shift is better than the linear one. The phase shift derived by fitting the modulated fraction is $281.8 \pm 44.6$ days, corresponding in phase to $\sim 0.2$ of the $1667 \pm 8$ day super-orbital period. The phase shift derived by fitting the maximum flux is $300.1 \pm 39.1$ days, which are compatible with the former.

This super-orbital modulation is also reported in Chernyakova et al. 2012. We show that there is a $\sim 0.2$ phase shift between the radio and the X-ray super-orbital modulation. Torres et al. 2012 has recently discussed that LS I +61 303 could be subject to a flip-flop behavior. The super-orbital modulation is possibly due to the cyclic change of the circumstellar disk (Li et al. 2012a; see also Papitto et al. 2012). In this context, multi-wavelength super-orbital modulation is expected and confirmed in radio, optical, X-ray and hinted in $\mathrm{GeV}$ and $\mathrm{TeV}$ ( $\mathrm{Li}$ et al. 2012a; Hadasch et al. 2012). 

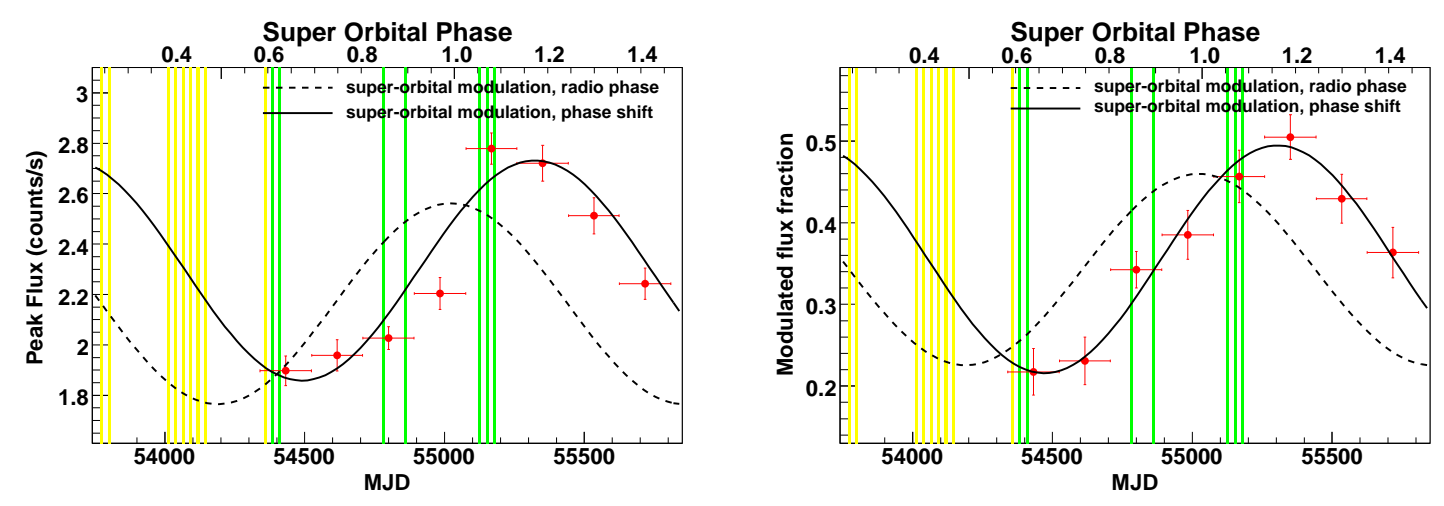

Figure 1: Left: Peak count rate of the X-ray emission from LS I +61 303 as a function of time and the super-orbital phase. Right: modulated fraction, see text for details. The dotted line shows the sine fitting to the modulated flux fraction and peak flux with a period and phase fixed at the radio parameters (from Gregory 2002). The solid curve stands for sinusoidal fit obtained by fixing the period at the 1667 days value, but letting the phase vary. The time bin corresponds to six months. The colored boxes represent the times of the $\mathrm{TeV}$ observations that covered the broadly-defined apastron region. The boxes in green denote the times when $\mathrm{TeV}$ observations are in low state while boxes in yellow are $\mathrm{TeV}$ observations in high state.

Table 1: Reduced $\chi^{2}$ for fitting different models to the modulation fraction and the peak flux in X-rays.

\begin{tabular}{lllll}
\hline & Constant & Linear & Radio & Shifted \\
\hline Modulation Fraction & 88.2 / 7 & $38.0 / 6$ & $42.1 / 6$ & $1.1 / 5$ \\
Peak Flux & $212.8 / 7$ & 114.8 / 6 & 91.8 / 6 & 4.9 / 5 \\
\hline
\end{tabular}

\section{INTEGRAL detection of 1FGL J1018.6-5856}

1FGL J1018.6-5856 is a newly discovered gamma-ray binary by Fermi/LAT. It has an orbital period of $16.58 \pm 0.04$ days and an epoch of maximum gamma-ray flux at MJD 55403.3 \pm 0.4 (Corbet et al. 2011). A coincident X-ray flux was found using Swift X-ray telescope (XRT) observations as well as a star of magnitude B2 which in turn coincides with the Swift/XRT detection. We present the results of the analysis of 5.78 Ms INTEGRAL/ISGRI data on the source 1FGL J1018.6-5856 (Li et al. 2011). The available INTEGRAL observations when 1FGL J1018.6-5856 had offset angle less than 14, adding up to a total exposure time of 5.78 Ms. Our data set covers from 2003 January 11 to 2009 November 20 . An INTEGRAL detection of 1FGL J1018.6-5856 is derived by combining all the INTEGRAL/ISGRI data, with a significance level of $5.4 \sigma$ and an average intensity of 0.074 counts/s in the $18-40 \mathrm{keV}$ band (see Figure 2, left panel). We only obtain $1.63 \sigma$ in the $40-100 \mathrm{keV}$. We combine the images from different $\mathrm{ScWs}$ based on orbital phase bins and produce an orbital light curve. We show that the INTEGRAL orbital light curve of 1FGL J1018.6-5856 together with the Fermi/LAT periodicity in Figure 2, right panel. It hints a trend of having an anti-correlation between the hard X-ray emission and the Fermi/LAT periodicity. This is in line with the results for LS 5039 (see, e.g., Hoffmann et al. 2009) where the hard X-ray emission as measured with INTEGRAL is fully anti-correlated with the GeV emission as measured by Fermi/LAT (Abdo et al. 2009)-emphasizing a possible physical similarity of the two sources. However, the scarcity of counts makes it difficult to have a definitive proof of the variability and anti-correlation: a constant 

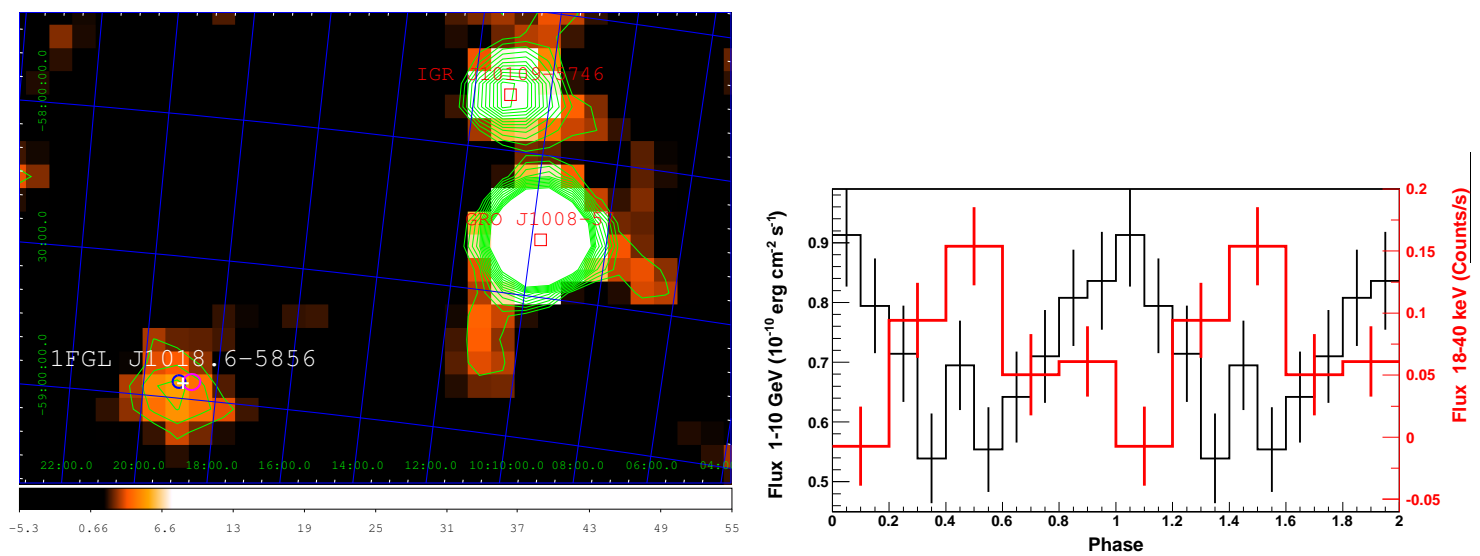

Figure 2: Left: Mosaic image of the 1FGL J1018.6-5856 sky region in the 18-40 keV band. The strongest source is GRO J1008-57 whereas the faintest one visible only in the image is 1FGL J1018.6-5856 The significance level is given by the color scale, with the contours starting at $3 \sigma$, and following steps of $1 \sigma$. The position of 1FGL J1018.6-5856 (magenta) as well as its updated center following the 2FGL FermiLAT Catalog is shown, while the cross represents the counterparts identified using XRT (from Corbet et al. 2011). Right: Orbital light curve of 1FGL J1018.6-5856 by INTEGRAL/ISGRI in 18-40 keV (red) and by Fermi/LAT in 1-10 GeV (black)

fit to the count rate yields a reduced $\chi^{2}$ of $14.09 / 4$, suggesting that the significance of variability is at only $2.7 \sigma$ level.

\section{INTEGRAL source $4 \mathrm{U} 1036-56$ as a possible gamma-ray transient}

In recent years, a number of unidentified, transient gamma-ray sources were discovered in the Galactic plane, especially by AGILE. Many of them have also been suggested to have a possible binary nature (see, Table 1 in Li et al. 2012b). All candidates have been suggested to have a possible high mass X-ray binary (HMXB) counterpart. Two gamma-ray transients (AGL J1037-5708 and GRO J1036-55) are located in the same region of the sky and spatially associated with HMXB 4U 1036-56 (see Figure 3). 4U 1036-56 is identified with a B0 III-Ve star LS 1698 at 5 kpc (Motch et al. 1997) and hosting a pulsar with a period of $853.4 \pm 0.2$ (La Palombara et al. 2009). We report on long-term analysis of INTEGRAL data on $4 \mathrm{U}$ 1036-56, examining the possibility of its association with the unidentified transient $\gamma$-ray sources AGL J1037-5708 \& GRO J1036-55 (Li et al. 2012b).

For the INTEGRAL analysis in this paper, we use all public IBIS/ISGRI and JEM-X data for which 4U 1036-56 has offset angle less than $9^{\circ}$ and $5^{\circ}$, respectively. The data covers from 2003 January to 2009 November, adding up to a total exposure time of $4.42 \mathrm{Ms}$ for IBIS/ISGRI and $1.02 \mathrm{Ms}$ for JEM-X . 4U 1036-56 is detected by IBIS/ISGRI with a significance level of $11.2 \sigma$ and an average intensity of $0.180 \pm 0.016$ counts s${ }^{-1}$ in the $18-60 \mathrm{keV}$ band. Figure 3 shows the IBIS/ISGRI and JEM-X mosaic image of the 4U 1036-56 sky region. 4U 1036-56 is detected by JEM-X at 3-20 keV at a significance of $4 \sigma$, with an average intensity of $0.88 \pm 0.22 \times 10^{-4}$ counts $/ \mathrm{cm}^{2} / \mathrm{s}$. 

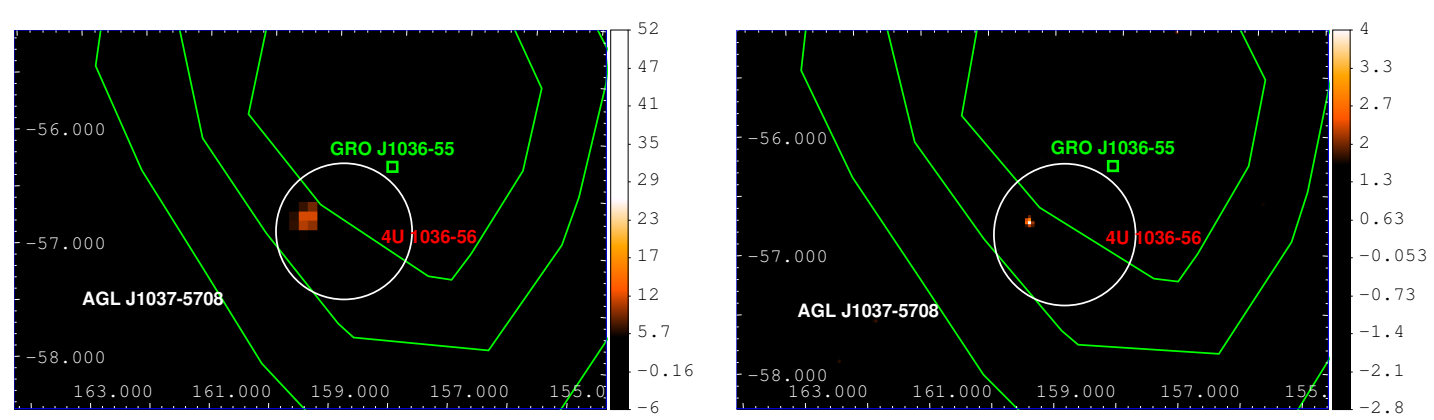

Figure 3: Mosaic image of the $4 \mathrm{U}$ 1036-56 sky region, derived by combining all IBIS/ISGRI data (18-60 $\mathrm{keV}$, left panel) and combining all JEM-X data (3-20 keV, right panel). The position of the transient AGILE source AGL J1037-5708 is plotted with its 95\% error region (white). Another transient source, GRO J103655 is shown in sky region with its $1,2,3 \sigma$ uncertainty location (green). The significance level is given by the color scale. Corresponding significance and color can be found in the right color bar. The $\mathrm{X}$-and $\mathrm{Y}$-axis are RA and Dec. in units of degrees.

We investigated the IBIS/ISGRI long-term light curve of 4U 1036-56 on the ScW timescale in the $18-60 \mathrm{keV}$ band. During most of the time, $4 \mathrm{U}$ 1036-56 is not significantly detected by IBIS/ISGRI . However, an outburst is discovered by IBIS/ISGRI between MJD 54142 (2007 Feb. 11) and MJD 54147 (2007 Feb. 16). This outburst at MJD 54144 is significantly detected by IBIS/ISGRI having a significance of $30.4 \sigma$ and an average intensity of $2.589 \pm 0.085$ counts s$^{-1}$ in the 18-60 keV band, over a $199 \mathrm{ks}$ exposure ( $\sim 1 / 22$ of the total exposure on the source). The JEM-X detection in the outburst period is significantly made at $10.1 \sigma$ with an average intensity of $0.194 \pm 0.019 \times 10^{-2}$ counts $/ \mathrm{cm}^{2} / \mathrm{s}$ in the $3-20 \mathrm{keV}$ band. Out of the outburst period, $4 \mathrm{U} 1036-56$ is detected by IBIS/ISGRI only with a significance of $5.7 \sigma$ under a total exposure time of $4.4 \mathrm{Ms}$ in the $18-60 \mathrm{keV}$ band. The average flux is $0.094 \pm 0.018$ counts s$^{-1}$ in the $18-60 \mathrm{keV}$ band (27 times dimmer than in outburst). JEM-X does not detect $4 \mathrm{U}$ 1036-56 during quiescence, yielding only $2.58 \sigma$ in the $3-20 \mathrm{keV}$, under a total exposure of $0.99 \mathrm{Ms}$. We extract an energy spectrum both from JEM-X and IBIS/ISGRI and fit with absorbed blackbody, an absorbed powerlaw, and an absorbed cutoff powerlaw model. We have found that only an absorbed cutoff powerlaw model could yield an acceptable fit. According to an F-test, the probability of refusing the cutoff to a simple power-law is $4.78 \times 10^{-4}$, corresponding to a significance of $3.5 \sigma$. Based on the spectrum parameters of the INTEGRAL outburst and assuming a source distance of $5 \mathrm{kpc}$ (Motch et al. 1997), the luminosity derived in $2-10 \mathrm{keV}$ band is $5.16_{-0.63}^{+0.72} \times 10^{35} \mathrm{erg} \mathrm{s}^{-1}$.

The positional coincidence as well as the variability timescales make it possible to entertain the hypothesis that the high-energy transients and 4U 1036-56 are related. We have shown that that it is theoretically feasible that HMXBs produce gamma-ray emission during periods of X-ray activity induced by accretion ( $\mathrm{Li}$ et al. 2012). Hence it is in principle plausible that $4 \mathrm{U}$ 1036-56 and the AGILE flares are related.

\section{INTEGRAL non-detection of HESS J0632+057 and AGL J2241+4454}

HESS J0632+057 is a gamma-ray binary with 321 days orbital periods (Bongiorno et al. 2011) and a companion of B0 Vpe star (Casares et al. 2011). It is detected in radio (Skilton et al. 2009), 
soft X-ray (Falcone et al. 2010) and TeV (Aharonian et al. 2007). We look for its hard X-ray counterpart with INTEGRAL. At an offset angle of 14 degree, HESS J0632+057 is observed by IBIS/ISGRI with a exposure of $0.81 \mathrm{Ms}$ and by JEM-X with $15 \mathrm{ks}$ exposure from 2003 to 2011. But HESS J0632+057 is not detected in the 18-60 keV energy range by IBIS/ISGRI nor in 3-35 $\mathrm{keV}$ range by JEM-X . AGL J2241+4454 is a transient gamma-ray source detected by the AGILE satellite above $100 \mathrm{MeV}$ (Lucarelli et al. 2010). It is possibly associated with Be star MWC 656 with an orbital period of $60.37 \pm 0.04$ days (Williams et al. 2010). It is a gamma-ray binary candidates ( $\mathrm{Li}$ et al. 2012b). We try to identified its counterpart in hard X-ray by INTEGRAL. The available INTEGRAL/ISGRI data on AGL J2241+4454 with an offset angle of 14 degree leads to a total exposure of $2.1 \mathrm{Ms}$. The detection significance is only 1.37 sigma in $18-60 \mathrm{keV}$. As a result, we do not have a detection of AGL J2241+4454 .

This work is supported by 973 program 2009CB824800 and the National Natural Science Foundation of China via NSFC-11233003, 11103020, 11133002, 11073021 and 11173023. Research done in the framework of the grants AYA2012-39303, AYA2009-07391, SGR2009-811, and iLINK2011-0303. We acknowledge Nanda Rea, Daniela Hadasch, Andrea Caliandro, Alessandro Papitto, and Yupeng Chen for joint work on these sources.

\section{References}

[1] Abdo, A. A., Ackermann, M., Ajello, M., et al., 2009, ApJ, 706, L56

[2] Aharonian, F. A. et al., 2007, A\&A, 469, L1

[3] Bongiorno, S. D., Falcone, A. D., Stroh, M., et al., 2011, ApJ, 737, L11

[4] Casares, J., Ribó, M., Ribas, I., et al., 2012, MNRAS, 421, 1103

[5] Chernyakova, M., Neronov, A., Molkov, S., et al., 2012, ApJ, 747, 29

[6] Corbet, R. H. D., Cheung, C. C., Kerr, M., et al., 2011, ATel, 3221

[7] Falcone, A. D., Grube, J., Hinto,n J.,et al., 2010, ApJ, 708, L52

[8] Gregory, P. C., 2002,ApJ, 575, 427

[9] Hadasch, D., Torres, D. F., Tanaka, T., et al., 2012, ApJ, 749, 54

[10] Hoffmann, A. D., Klochkov, D., Santangelo, A., et al., 2009, A\&A, 494, L37

[11] La Palombara, N., Sidoli, L., Esposito, P., Tiengo, A., \& Mereghetti, S., 2009,ApJ, 505, 947

[12] Li, J., Torres, D. F., Zhang, S., et al., 2012a,ApJ, 744, 13

[13] Li, J., Torres, D. F., Zhang, S., et al., 2012b,ApJ, 761, 49

[14] Li, J., Torres, D. F., Zhang, S., et al., 2011,ApJ, 733, 89

[15] Lucarelli F. et al., 2010, ATel, 2761

[16] Motch, C., Haberl, F., Dennerl, K., Pakull, M.,\&Janot-Pacheco, E., 1997, A\&A, 323, 853

[17] Skilton, J. L. et al., 2009, MNRAS, 399, 317

[18] Torres, D. F., et al., 2012,ApJ, 744, 106

[19] Papitto, A., Torres, D. F. \& Rea, N., 2012, ApJ, 756, 188

[20] Williams, S. J., Gies D. R., Matson, R. A., et al., 2010, ApJ, 723, L93 